\title{
Technology of Decreasing Injection Pressure and Increasing Injection Rate by Surfactant in CS block of Chaoyanggou Oilfield

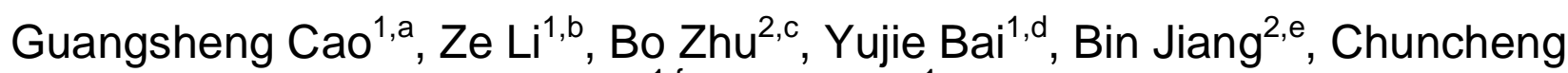 $\mathrm{Li}^{1, \mathrm{f}}$, Tianmin $\mathrm{Li}^{1, \mathrm{~g}}$
}

${ }^{1}$ Key Laboratory of Enhanced Oil and Gas Recovery Ministry of Education, Northeast Petroleum University, Daqing China, 163318;

${ }^{2}$ The tenth production plant of Daqing oil field co., LTD,Daqing China, 163114

acaoguangsheng@163.com, ${ }^{\mathrm{b}} 458599205 @ q q . c o m,{ }^{\mathrm{c}}$ zhubo0001@sina.com,

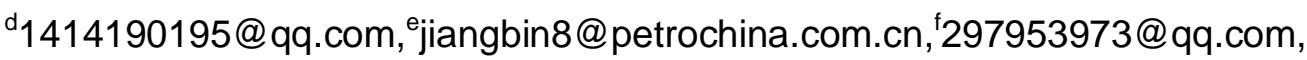
g349610362@qq.com

Keywords: The CS block of Chaoyanggou oilfield, Extra low permeable reservoir, Surfactant, Decreasing injection pressure.

Abstract: The CS block of Chaoyanggou oilfield is a extra low-permeability reservoir. In the process of water flooding, some problems which influence exploitation effect are commonly found, such as high flooding pressure and insufficient injection in water wells. Based on the analysis of lithology and physical property of CS block, the formula were obtained by measuring the IFT of oil and surfactant solution. According to the core simulation experiments, the injection parameters were optimized. Results indicate that the IFT of the CS block oil and formula system is $8.16 \times 10^{-3} \mathrm{mN} / \mathrm{m}$, the best concentration of surfactant is $0.5 \%$, the best injection volume is $0.5 \mathrm{PV}$, the ratio of decreasing injecting pressure is $38.90 \%$.

\section{Introduction}

The CS block of Chaoyanggou Oilfield is a extra low-permeability reservoir. In a extra low-permeability reservoir, the void construction is complex, exploitation characteristics are different from middle and high permeability reservoirs. Problems such as high flooding pressure, insufficient injection rate and bad exploitation effect are commonly found. Research indicates that, by using surfactant solution, the capillary pressure of water flooding and the IFT of oil and water can be reduced, the mobility of oil can be promoted, the injecting pressure can be reduced ${ }^{[1]}$. Surfactant flooding can decrease the injection pressure of low permeability cores, increase the permeability of both phases, and improve the EOR efficiently. Detection results exhibit that with the decrease of the IFT of oil and water and the increase of the surfactant slug size, the effectiveness of lowering injection pressure rises. There is a best parameter combination in surfactant flooding ${ }^{[2]}$.

Based on the exploitation conditions and the geologic characteristic, this paper studied a formula of surfactant for decreasing injection pressure and increasing injection rate. According to the core displacement experiment, the range of decreasing injecting pressure is evaluated. Finally, the best parameter combination which fits the CS block is obtained.

\section{Basic features of reservoir in CS block}

The lithology of blocks in Fuyu reservoir is mainly lithic arkose. The degree of sorting is medium.

The composition of clay mineral is mainly goeschwitzite, the average content is $44.4 \%$; the second 
adglutinate is chlorite and kaolinite, the average content is $34.4 \% \%$ and $14.4 \%$ respectively.

According to the analysis of core-taking from two wells in this block, the average of effective porosity and permeability in the CS block is $16.7 \%$ and $5.2 \times 10^{-3} \mu \mathrm{m}^{2}$ respectively. The CS block is a low-porosity and extra low-permeability reservoir.

\section{Development of surfactant system}

By reducing the IFT of oil and water, the surfactant can attain the result of decreasing injection pressure and increasing injection rate. With the decreasing of IFT, the ratio of decreasing injecting pressure will be increasing. With the increasing of the permeability, the ratio of decreasing injecting pressure will be increasing ${ }^{[3]}$. In order to ensure the effect of decreasing injection pressure and increasing injection rate in water injection wells, the selected surfactant must have lower IFT. By using the surfactant, the IFT of oil and water can be reduced extremely, the wettability of the surface of rock can be changed, the acting force among the phases can be reduced, the result of decreasing injection pressure and increasing injection rate can be obtained ${ }^{[4]}$.

Therefore, choosing the surfactant by measuring the IFT, mixing the selected the surfactants with relatively low IFT and adding additives, finally, a surfactant formula for decreasing injection pressure and increasing injection rate is obtained. Making up mixture solution $0.5 \%$ which compounded with various surfactant,then, the interfacial tension of mixture solution and oil were measured in the $60^{\circ} \mathrm{C}$ by interfacial tensiometer (TX-500D).

Main agent and adjuvant. Measuring the IFT between the CS block oil and 8 kinds of surfactants, the result is shown in Table 1.

Table1 the IFT between oil and single surfactant

\begin{tabular}{|c|c|c|c|c|}
\hline Number & $\begin{array}{c}\text { Surfactant } \\
\text { NO. }\end{array}$ & Type & $\begin{array}{c}\text { Concentration } \\
(\%)\end{array}$ & IFT $(\mathrm{mN} / \mathrm{m})$ \\
\hline 1 & $\mathrm{SC}-1$ & anion -mahogany sulfonate & 0.5 & $2.71 \times 10^{-1}$ \\
\hline 2 & ZWS-DQ & anion -HABS & 0.5 & 9.28 \\
\hline 3 & SDBS & anion -ABS & 0.5 & 5.52 \\
\hline 4 & SDSS & anion -alkyl sulfate & 0.5 & 11.96 \\
\hline 5 & JYM & $\begin{array}{c}\text { nonionic-fatty alcohol } \\
\text { polyether }\end{array}$ & 0.5 & $8.19 \times 10^{-1}$ \\
\hline 6 & DYXY-4 & nonionic-alkylphenol ether & 0.5 & $2.77 \times 10^{-1}$ \\
\hline 7 & DYF-SH & $\begin{array}{c}\text { nonionic -fluorocarbon } \\
\text { polyether }\end{array}$ & 0.5 & $1.65 \times 10^{-3}$ \\
\hline 8 & DYSiF & nonionic -anion complex & 0.5 & $7.92 \times 10^{-1}$ \\
\hline
\end{tabular}

Table 1 illustrates the IFT difference between the CS block oil and 8 kinds of surfactants is tremendous. Only DYF-SH (nonionic -fluorocarbon polyether) is in the magnitude of $10^{-3} \mathrm{mN} / \mathrm{m}$. Therefore, DYF-SH (nonionic -fluorocarbon polyether) is selected as main agent. On account of the price of DYF-SH is high, hence, DYF-SH is compounded with other surfactants in a ratio 4:1.Only the IFT of compound of SC-1 and DYF-SH attains the magnitude of $10^{-2} \mathrm{mN} / \mathrm{m}$. Therefore, $\mathrm{SC}-1$ (anion -mahogany sulfonate) is selected as adjuvant.

Additives. Alcohol and ether compounds can decrease IFT. 7 kinds of additives were selected to 
add into the mix solution of SC-1 $(0.4 \%)$ and DYF-SH(0.05\%), the effect of adding DCN-4 is best, the IFT is $1.30 \times 10^{-2} \mathrm{mN} / \mathrm{m}$, but does not attain the requirement of the magnitude of $10^{-3} \mathrm{mN} / \mathrm{m}$.

Formula. In order to decrease IFT further, the concentration of main agent, adjuvant and additive is optimized. The result is shown in Table 2 .

Table2 The optimization of the concentration of chemicals

\begin{tabular}{c|c|c|c|c}
\hline Number & SC-1 $(\%)$ & DYF-SH $(\%)$ & DCN-4 $(\%)$ & IFT $(\mathrm{mN} / \mathrm{m})$ \\
\hline 1 & 0.4 & 0.03 & 0.07 & $7.95 \times 10^{-2}$ \\
\hline 2 & 0.4 & 0.05 & 0.05 & $1.30 \times 10^{-2}$ \\
\hline 3 & 0.4 & 0.07 & 0.03 & $8.16 \times 10^{-3}$ \\
\hline 4 & 0.4 & 0.09 & 0.01 & $9.91 \times 10^{-3}$ \\
\hline 5 & 0.3 & 0.1 & 0.1 & $2.84 \times 10^{-2}$ \\
\hline 6 & 0.3 & 0.05 & 0.15 & $9.28 \times 10^{-3}$ \\
\hline 7 & 0.35 & 0.1 & 0.05 & $4.09 \times 10^{-2}$ \\
\hline 8 & 0.35 & 0.05 & 0.1 & $0.105 \mathrm{~m} / \mathrm{m}$ \\
\hline
\end{tabular}

Table 2 illustrates three formulas attain the requirement of magnitude of $10^{-3} \mathrm{mN} / \mathrm{m}$. Concerning the effect of decreasing pressure and cost synthetically, the best formula is obtained: $0.4 \% \mathrm{SC}-1+$ $0.07 \%$ DYF-SH $+0.03 \%$ DCN-4 (IFT is $8.16 \times 10^{-3} \mathrm{mN} / \mathrm{m}$ ).

\section{Experiments}

In different concentration and dosage of surfactant conditions, the surfactants were injected into cores in displacing oil experiment. The best concentration and volume are attained. Parameters of natural core are shown in Table 3.

Table 3 Parameters of natural core

\begin{tabular}{c|c|c|c|c}
\hline Number & Length $(\mathrm{cm})$ & Diameter $(\mathrm{cm})$ & $\begin{array}{c}\text { Permeability } \\
\left(10^{-3} \mu \mathrm{m}^{2}\right)\end{array}$ & Porosity (\%) \\
\hline CS-1 & 7.90 & 2.56 & 3.9 & 10.3 \\
\hline CS-2 & 8.61 & 2.49 & 7.6 & 15.7 \\
\hline CS-3 & 7.4 & 2.52 & 4.5 & 12.9 \\
\hline CS-4 & 8.1 & 2.49 & 8.3 & 14.6 \\
\hline CS-5 & 8.3 & 2.48 & 6.5 & 13.3 \\
\hline CS-6 & 7.8 & 2.51 & 4.9 & 15.9 \\
\hline CS-7 & 8.4 & 2.50 & 7.8 & \\
\hline
\end{tabular}

The influence of surfactant concentration on injecting pressure. Because surfactant may be adsorbed by natural core, the formula obtained above must be optimized further by core displacement experiment. The natural cores (From CS-1 to CS-4) were saturated with formation water and then saturated with oil. In the condition of constant rate of $0.5 \mathrm{ml} / \mathrm{min}$, injecting water process was simulated. When injecting water pressure reached the maximum value, surfactant solutions with 4 kinds of concentration(refer with Table 4) were injected 1PV into these cores respectively, then water was injected too. The change of injecting pressure is shown in Fig.1.

Table 4 Composition of formula

\begin{tabular}{c|c|c|c}
\hline Concentration of formula (\%) & SC-1 (\%) & DYF-SH (\%) & DCN-4 (\%) \\
\hline 0.3 & 0.24 & 0.042 & 0.018 \\
\hline 0.5 & 0.4 & 0.07 & 0.03 \\
\hline 0.7 & 0.5 & 0.098 & 0.042 \\
\hline 1.0 & 0.7 & 0.14 & 0.06 \\
\hline
\end{tabular}




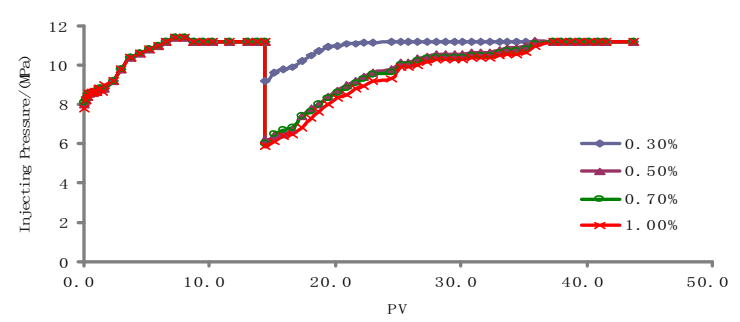

Fig.1 The influence of surfactant concentration on injecting pressure

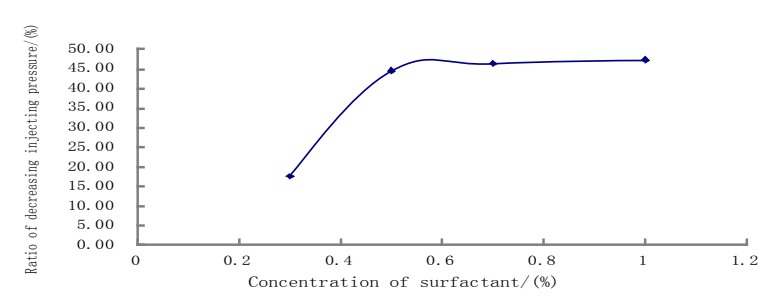

Fig.2 The relation curve of the ratio of decreasing pressure and concentration

Fig. 1 illustrates that, when the concentration of surfactant solution is $0.3 \%, 0.5 \%$ and $1.0 \%$, the ratio of decreasing pressure is $17.66 \%, 44.56 \%$ and $47.31 \%$ respectively.

Fig.2 illustrates that, with the injecting surfactant concentration increasing, the ratio of decreasing pressure will be increasing. When the injecting concentration exceed $0.5 \%$, with the injecting surfactant concentration increasing, the ratio of decreasing pressure will change slightly. Therefore, the best concentration is $0.5 \%$, its ratio of decreasing pressure is $44.56 \%$.

The influence of slug volume of surfactant solution on injecting pressure. The natural cores (From CS-1 to CS-4) were saturated with formation water and then saturated with oil. In the condition of constant rate of $0.5 \mathrm{ml} / \mathrm{min}$, injecting water process was simulated. When injecting water pressure reached the maximum value, $0.3 \mathrm{PV}, 0.5 \mathrm{PV}$ and $1.0 \mathrm{PV}$ of surfactant solutions(concentration: $0.5 \%$ ) were injected into these cores respectively, then water was injected too. The change of injecting pressure is shown in Fig.3.

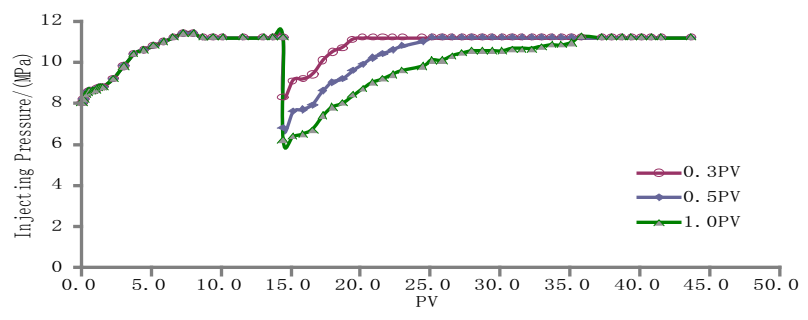

Fig.3 The figure of change of injecting pressure of different injecting PV of surfactant

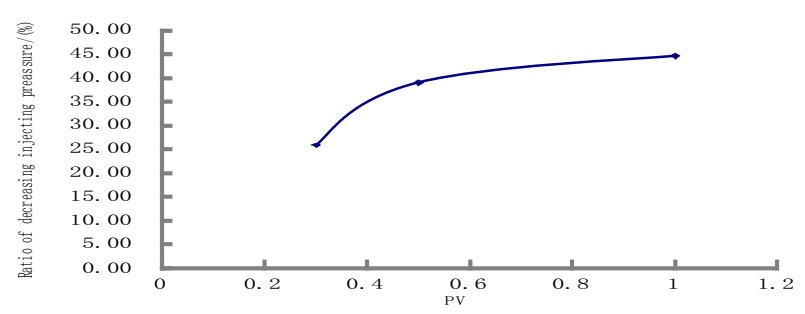

Fig.4 The relation curve of the ratio of decreasing pressure and PV of surfactant

Fig.3 illustrates that, when injecting surfactant solution of $0.3 \mathrm{PV}, 0.5 \mathrm{PV}$ and $1.0 \mathrm{PV}$, the ratio of decreasing pressure is $25.81 \%, 38.90 \%$ and $44.56 \%$ respectively.

Fig.4 illustrates that, as the volume of surfactant solution increasing, the ratio of decreasing pressure will increase. When the injected volume exceed $0.5 \mathrm{PV}$, the ratio of decreasing pressure will change slightly. Concerning the economic cost, therefore, the best volume is $0.5 \mathrm{PV}$, the ratio of decreasing injecting pressure is $38.90 \%$ correspondingly.

\section{Conclusions}

(1) According to the reservoir characters of Cs block in Chaoyanggou oilfield, the surfactant formula for decreasing injection pressure and increasing injection rate is obtained: $0.4 \% \mathrm{SC}-1+0.07 \% \mathrm{DYF}-\mathrm{SH}+0.03 \% \mathrm{DCN}-4$, the IFT of formula and the CS block oil is $8.16 \times 10^{-3} \mathrm{mN} / \mathrm{m}$.

(2)The best parameter combination: the injecting concentration of surfactant is $0.5 \%$, the injecting dosage of surfactant is $0.5 \mathrm{PV}$, The ratio of decreasing injecting pressure is $38.90 \%$ correspondingly. 


\section{References}

[1] Yaning Liang, "Technology of Decreasing injection Pressure and Increasing Injection Rate by Surfactant in the Daqing Oilfield", in Chinese, Daqing Petroleum Institute Master Degree Thesis, 2006

[2] Qian Wang, "Experimental Study of Surfactant Flooding in Reducing Injecting Pressure and EOR for Low-permeability Reservoir", in Chinese, China University of Petroleum Master Degree Thesis, 2015

[3] Guiguan Wang, Aifang Li, Yongzheng Wang, etc, Science Technology and Engineering, Vol.15,issue 6 (2015),1671-1815

[4] Zengbao Wang, Jing Wang, Ming Tong, etc., Oilfield Chemistry, Vol. 31,issue 4(2014),573-577 